\title{
EFEITO DA INTERFERENCIA DE DESLIZAMENTO NA MOBILIDADE DENTAL
}

\section{(The effect of sliding interference on tooth mobility)}

\author{
JOSÉ D. FREITAS VALE, C.D., M.S. * \\ EZIO TESEO MAINIERI, C.D. **
}

A modified version of the Mühlemann macroperiodontometer was used to study horizontal tooth mobility in 36 human subjects with sliding interferences. It was found an increased horizontal tooth mobility in the group of teeth which received the impact at the end of the slide in centric as compared to the control group. However, the physiologic tooth mobility limits were not exceeded. The results demonstrated a great variation in horizontal tooth mobility values for specific teeth.

\section{INTRODUÇAO}

A mobilidade dental é um parâmetro de reconhecida importância, tanto clínica quanto cientificamente. Seu significado é óbvio quando se considerar que mobilidade dental $\dot{\epsilon}$ um sinal de integridade, disfunção od doença do periodontium.

Investigações clínicas realizadas têm demonstrado que a mobilidade horizontal dos dentes é maior pela manhã decrescendo progressivamente até à noite, o que pode ser correlacionado com a leve intrusão dos mesmos nos seus alvéolos devidos às forças oclusais 13. Opostamente, se os dentes forem mantidos afastados por um período de tempo, a mobilidade aumenta, talvez por um mecanismo inverso ao anterior, qual seja, uma leve extrusão 14 . Contudo, presumese que os contatos dentais, durante 2 deglutição, são suficientes para man. ter a moiblidade dental horizontal em niveis normais, ainda que năo haia contatos oclusais mastigatórios 15 .

No entanto, mais pesquisa é necessária para avaliar o feito do sexo e idade sobre a mobilidade fisiológica, embora pareça que as mulheres apreseniem maior mobilidade dental do que os homens $e$ os idosos menos mobilidade fisiológica do que os ju. vens. Além disso, sabe-se que a gra videz leva a um aumento da mesma 19.

Entre os fatores locais que afetam a mobilidade horizontal de urn dente, as forças oclusais anormais contribuem decisivamente para o se' aumento. O bruxismo é comumente associado a essa situaçåo, especial-. mente quando o aumento da mobilidade é encontrado em dentes sem evidência de moléstia periodontal, reabsorção radicular e problemas pe. riapicais 5 .

Por outro lado, há uma correlaçüo positiva entre a perda de tecido de suporte periodontal e grau de mobilidade dental 10, entretanto uma mastigação vigorosa pode diminuir a mo-

\footnotetext{
* Disciplina de Periodontia.

** Disciplina de Oclusão. - Departamento de Odontologla Conservadora. - Faculdade de Odontologia, Universidade Federal do Rio Grande do Sul - Brasil.
} 
bilidade ou mesmo não interferir nela 16. Já durante o tratamento ortodôntico é evidente o fenômeno hipermobilidade dental 9 .

Resumindo, o grau de mobilidacio de um dente depende de forças aplicadas nele e a resistência das estristuras de suporte. E necessário considerar a or:gem da força, a direção, a magnitude e a frequência; a resistência envolve a extensão das áreas de suporte e a integridade dos tecidos de suporte. A integridade' dos tecidos de suporte pode ser alterada por trauma da oclusão por perda da suporte devido à moléstia periodontal, por condições blastomatóides e neoplásicas e por condições sistêmicas que alteram a polimerização da substância fundamental e afetam a integridade das fibras colágenas da membrana periodontal 18.

Em 1951, Muhlemann introduziu a periodontometria 8 , e desde então uma série de dispositivos mecânicos e eletrônicos tem sido empregada para testar cientificamente a mobilidade dental tanto horizontal 10,12 quanto verticalmente 17.

O objetivo desta investigação é determinar se a mobilidade dental horizontal pode ser aumentada em consequência das forças oclusais provonientes do impacto no final do desizamento em cêntrica.

\section{MATERIAL E MÉTODOS}

A pesquisa foi realizada em 36 alunos da F.O.U.F.R.G.S., sendo 17 do sexo feminino e 19 do sexo masculino, com idade variando entre 19 e 27 anos.
Com o fim de eliminar-se uma série de variáveis, várias restrições foram colocadas para a aceitação dos participantes do estudo, a saber:

1. - Paciente não deve ter portado ou ser portador de próteses fixas ou removiveis, nem ter história recente de tratamento ortodôntico.

2. - Paciente năo deve apresentar sinais ou sintomas de disfun. ção do Sistema Mastigatório (bruxismo, facetas de desgaste, etc...).

3. - Paciente deve apresentar seus tecidos periodontais, tanto marginal quanto apical com saúde.

4. - Paciente não deve ter história positiva de fatores sistêmicos, tanto fisiológicos como patológicos.

5. - Paciente deve ter ao menos dois dentes de cada grupo em oposição em oclusão cêntrica em ambas as arcadas.

6. - Paciente deve apresentar mastigação bilateral.

7. - Paciente não deve ter seus dentes, cujas mobilidades horizontais serão medidas, envolvidos em prematuridades em relação e oclusão cêntricas e/ou em interferências nos movimentos excursivos.

8. - Paciente deve ter seus dentes, cujas mobilidades horizontais serão medidas, com antagoniśtas.

O exame dos pacientes teve inicio com a identificação das prematuridades em relação cêntrica, oclusão cênírica e interferências excursivas além do registro dos dentes envoly: 
dos no final do deslizamento em cên trica. Para tanto foi utilizado o papel articular.

As prematuridades e interperências foram classificadas pelos seguintes critérios:

1. - Prematuridade em oclusão cèntrica.

O paciente foi instruído para tocar seus dentes repetidas vezes em máxima intercuspidação. Qualquer dente que apresentasse contato prematuro consistente era anotado como prematuridade em oclusão cêntrica.

2. - Prematuridade em relação cêntrica.

A mandíbula do paciente fui normalmente guiada para sua posição mais retrusiva, movi- mento de abertura e fechamento em bisagra. Os dentes que fizeram contato inicial durante 0 fechamento nesta posição foram registrados como prematuridade èm relação cêntrica.

3. - Interferência de deslizamento. Com a mandínbula do paciento em relação cêntrica $\theta$ com os dentes envolvidos no contato prematuro nessa posição se tocando instruiu-se o paciente no sentido de contrair sua musculatura elevadora da mandínbula O movimento mandibular que se seguiu foi chamado de deslizamento em cêntrica e os dentes que receberam o impacto no fi:nal do mesmo foram anotados como interferência de deslizamento. Vide Fig. 1
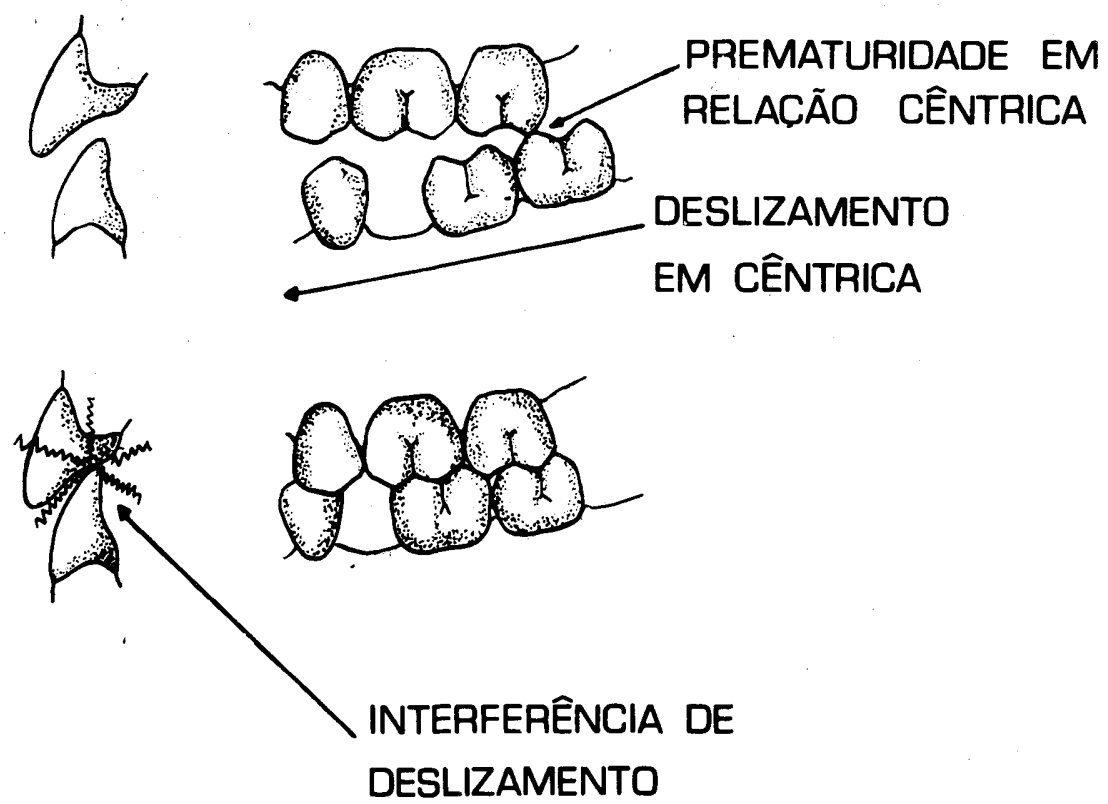

R. Fac. Odont. P. A. 
4. - Interferência no lado de tráoathó.

Da oclusão cêntrica o paciente foi instruido para mover sua mandibula lateralmente. Os derites do lado da arcada para onde - movimento foi executado $e$ que impediram um moviments suave foram anotados como in. terferências de trabalho. Isso foi feito para os lados direito e es querdo.

5. - Interferências no lado de balan. ceio.

Novamente, da posição de ocl:são cêntrica e paciente foi instruído para mover sua mandíbula lateralmente. Os dentes que fizeram qualquer contato no lado oposto, ao de trabalho foram registrados como interferências de balenceio.

6. - Interferência protrusiva.

Da oclusão cêntrica o paciente foi instruído para mover sua mandibula diretamente para a frente. Os dentes.que impediram um movimento suave foram anotados como interferências protrusivas.

Fe to o exame da oclusão de cada paciente, com a finalidade de seleção, tratou-se de medir as mobilidades horizontais dos dentes envolvidos nas interferências de deslizamento e dos seus homólogos do lado oposio (controle). Assim, de cada paciente mediram-se as mobilidades horizontais de quatro dentes. Todas as medições foram fe'tas às nove horas di manhã.

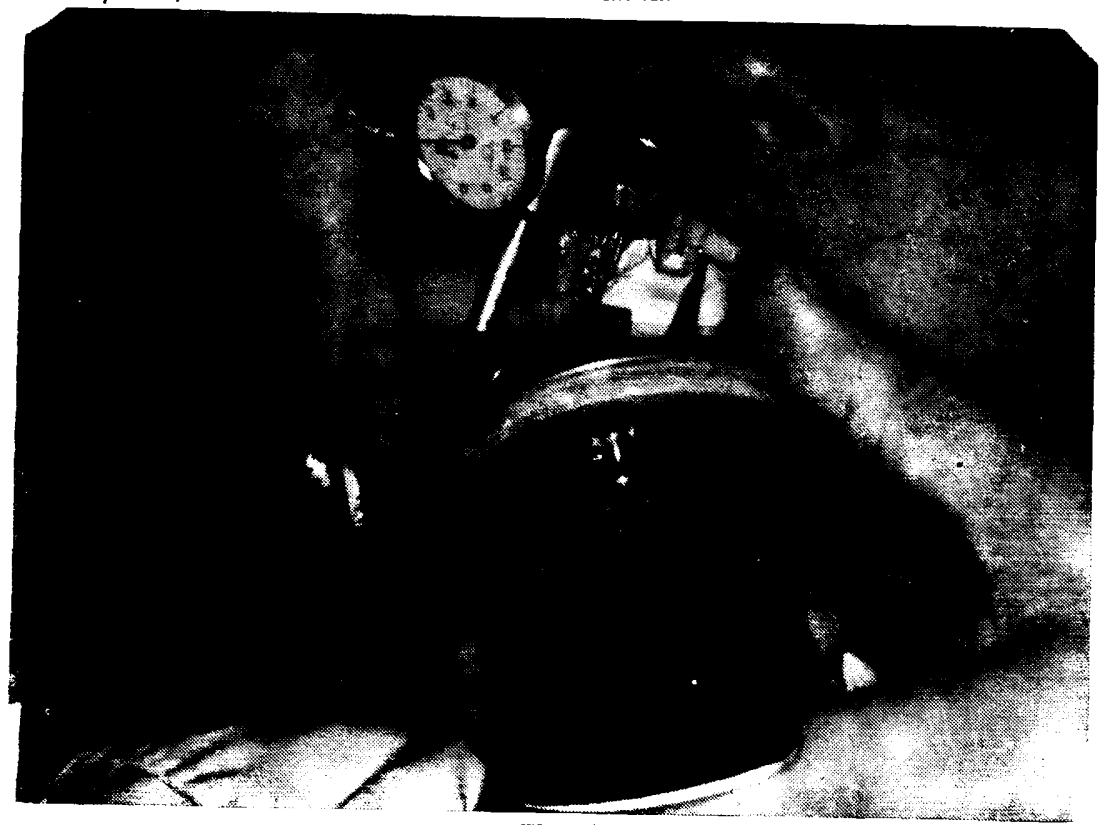

Fig. 2

R. Fac. Odont. P. A. 
O método de mediçăo utilizaço rizontais foram executadas, nos pre: ıi o de Mühlemann através do se'j macro-periodontômetro, o qual é sên. sível a movimentações de até um centésimo de milimetro. Houve a neces. sidade de se fazerem certas adapta. ções no aparelho para que, se pudessem medir as' mobilidades hoi. zontais dos dentes inferiores e segundos pré-molares superiores.

O aparelho foi montado numa moldeira de impressão e fixado la boca do paciente com gesso solúvel. A ponta indicadora do aparelho foi adaptada à face vestibular do dente superior que recebeu o impacto rio final do deslizamento em cêntrica (interferência de deslizamento).

A seguir, uma força de 500 griamas foi aplicada durante 3 segundos, por meio de um dinamômetro no sentido líinguo-vestibular, no dente em questão. Vide Fig. 2

O mesmo procedimento foi repetido, sucessivamente, para o dente in. ferior envolvido na interferência de deslizamento $\theta$ para os dentes homólogos do lado oposto (controle).

Com a finalldade de se conseg: rem resultados mais acurados, foram feitas 3 medições sucessivas em $c a$ da dente que teve sua mobilidade $h c$ rizontal registrada e a média obtica representava a mobilidade absoluta ds mesmo.

\section{RESULTADOS}

O levantamento das interferênclas de deslizamento revelou que a maior incidencia destas recaía sobre os prí molares.

As medições das mobilidades ho

R. Fac. Odont. P. A. molares envolvidos $\theta$ homologos (gri po con'role), tanto superiores qi.dr inferiores, em número de três part cada dente e a média obtida foi cir ciderada a representaçăo numérica dente em questão.

Tendo em vista que as mobilidades horizontais dos dentes envolvidos e homólogos, expressas em centési mos de milimetros $(\mathrm{cmm})$ eram ind $\theta$. pendentes, utilizou-se o teste de hipotese bilateral sobre a diferença das médias, conforme fórmulas que seguem:

$$
\begin{aligned}
& t=\frac{\left(\vec{x}_{A}-\vec{x}_{B}\right)}{v^{A} C^{2}} \frac{1}{n_{A}}+\frac{1}{n_{3}} \\
& U^{2}=\frac{n_{A} \hat{5}_{A}^{2}+n_{B} \hat{5}_{B}^{2}}{n_{A}+n_{B} \cdot 2}
\end{aligned}
$$

Considerando que a médla de mobilidade dos pré-molares superiores envolvidos era de 10,333, com uma variância de 1,722 e a média de $m o$ bilidade dos dentes homólogos 9,194, com uma variância de 1,657. E que os pré-molares inferiores envolvidos apresentavam uma média de mobilidade de 9,972, com uma variância de 1,027 , enquanto que seus homóloges apresentavam 8,778, com uma variâ.1cla de 0,951 , verificou-se que a diferença das médias era estatisticamenta significante, ao nivel de $1 \%$, uma vez que resultou $p<0,01$ tanto para os premolares superiores quanto para us pis molares inferiores (Tabelas 1 e II). 
$\begin{array}{lllllll}T & \text { A B } & \text { E L A } & \text { I }\end{array}$

MOBILIDADES DOS PRE-MOLARES SUPERIORES

\begin{tabular}{|c|c|c|c|}
\hline \multicolumn{2}{|c|}{$E N \vee O L L V I D O$} & \multicolumn{2}{|c|}{$H O M O L O G O$} \\
\hline DENTE & MOBILIDADE & DENTE & MOBILIDADE \\
\hline & $\mathrm{cmm}$ & & $\mathrm{cmm}$ \\
\hline 24 & 11 & 14 & 10 \\
\hline 25 & 9 & 15 & 8 \\
\hline 14 & 9 & 24 & 10 \\
\hline 25 & 10 & 15 & 8 \\
\hline 14 & 10 & 24 & 7 \\
\hline 15 & 9 & 25 & 9 \\
\hline 24 & 10 & 14 & 8 \\
\hline 24 & 13 & 14 & 10 \\
\hline 14 & 9 & 24 & 10 \\
\hline 25 & 10 & 15 & 8 \\
\hline 25 & 10 & 15 & 7 \\
\hline 15 & 8 & 25 & 8 \\
\hline 25 & 12 & 15 & 10 \\
\hline 24 & 11 & 14 & 8 \\
\hline 25 & 11 & 15 & 9 \\
\hline 25 & 8 & 15 & 8 \\
\hline 25 & 9 & 15 & 8 \\
\hline 24 & 12 & 14 & 10 \\
\hline 14 & 10 & 24 & 10 \\
\hline 25 & 10 & 15 & 11 \\
\hline 15 & 11 & 25 & 7 \\
\hline 24 & 11 & 14 & 10 \\
\hline 15 & 13 & 25 & 11 \\
\hline 24 & 10 & 14 & 11 \\
\hline 24 & 12 & 14 & 9 \\
\hline 24 & 11 & 14 & 9 \\
\hline 15 & 12 & 25 & 10 \\
\hline 24 & 10 & 14 & 9 \\
\hline 14 & 9 & 24 & 9 \\
\hline 24 & 12 & 14 & 12 \\
\hline 25 & 8 & 15 & $B$ \\
\hline 25 & 10 & 15 & 9 \\
\hline 24 & 11 & 14 & 11 \\
\hline 15 & 8 & 25 & 8 \\
\hline 25 & 11 & 15 & 10 \\
\hline 15 & 11 & 25 & 11 \\
\hline TOTAL & 372 & & 331 \\
\hline
\end{tabular}

R. Fac. Odont. P.A.

$15 / 16 / 17: 59-68,1974 / 75 / 7{ }^{\prime}$ 


\section{T A B E L A I I}

MOBILIDADES DOS PRÉ-MOLARES INFERIORES

\begin{tabular}{|c|c|c|c|}
\hline \multicolumn{4}{|c|}{$E N \vee O L V I D O$} \\
\hline DENTE & MOBILIDADE & DENTE & MOBILIDADE \\
\hline & $\mathrm{cmm}$ & & $\mathrm{cmm}-$ \\
\hline $34 \cdot$ & 9 & 44 & 9 \\
\hline 35 & 8 & 45 & 9 \\
\hline 44 & 8 & 34 & 8 \\
\hline 35 & 10 & 45 & 7 \\
\hline 44 & 9 & 34 & 8 \\
\hline 45 & 9 & 35 & 8 \\
\hline 35 & 9 & 45 & 9 \\
\hline 34 & 12 & 44 & 9 \\
\hline 44 & 10 & 34 & 10 \\
\hline 35 & 11 & 45 & 9 \\
\hline 35 & 9 & 45 & 7 \\
\hline 45 & 11 & 35 & 10 \\
\hline 35 & 11 & 45 & 10 \\
\hline 35 & 11 & 45 & 9 \\
\hline 35 & 10 & 45 & 8 \\
\hline 35 & 9 & 45 & 7 \\
\hline 35 & 9 & 45 & 8 \\
\hline 34 & 10 & 44 & 9 \\
\hline 44 & 9 & 34 & 8 \\
\hline 35 & 10 & 45 & 10 \\
\hline 45 & $1 \overline{10}$ & 35 & 8 \\
\hline 34 & 11 & 44 & 9 \\
\hline 35 & 11 & 45 & 7 \\
\hline 34 & 9 & 44 & 9 \\
\hline 34 & 11 & 44 & 8 \\
\hline 34 & 10 & 44 & 8 \\
\hline 45 & 11 & 35 & 10 \\
\hline 34 & 10 & 44 & 10 \\
\hline 44 & 10 & 34 & 9 \\
\hline 34 & 10 & 44 & 9 \\
\hline 35 & $\dot{9}$ & 45 & 9 \\
\hline 34 & 11 & 44 & 10 \\
\hline 34 & 11 & 44 & 10 \\
\hline 44 & 9 & 34 & 9 \\
\hline 35 & 10 & 45 & 10 \\
\hline 45 & 12 & 35 & 10 \\
\hline OOTAL & 359 & & 316 \\
\hline
\end{tabular}

R. Fac. Odont. P. A. 


\section{DISCUSSÃO}

Os resultados indicaram que as mobilidades horizontais dos dentes que receberam o impacto no final do deslizamento em cêntrica (interferênc:a de deslizamento) eram maiores do que as dos dentes controles. Este fato pode ser correlacionado com a deglutição, uma vez que se acredita que a mandíbula seja deslocada para distal durante a mesma 6 . Estudios com rádio transmissores 3,6 demonstraram que há contatos dentais regularmente com a mandibula em relação cêntrica durante a deglutição. A discrepância entre relação cêntrica e oclusão cêntrica pode envolver um deslizamento em cêntrica que é aquele em que a mandibula se move para a frente a partir de um contato inicial em relação cêntrica ou próximo dela.

Os dentes que foram catalogados como interferências de deslizamento sofreram o impacto ao final deste movimento e talvez a hipermobilidade horizontal apresentada por eles seja a expressão de uma situação traumática inevitável, considerando que o deslocamento da mandibula durante a deglutição seja parte de um padrão reflexo incondicionado primário e que os contatos oclusais são partes integrantes da deglutição.

Acrescente-se que a freqüência do ato de deglutir foi estabelecida em uma vez cada dois minutos 7 em pessoas acordadas, sendo, no entanro, menos frequente durante o sono, po:tanto haverá um impacto sobre a interferência de deslizamento com a mesma freqüência.
A intensidade das forças liberadas pelo impacto dependerá do tônus muscular individual, o que é, por sua vez, influenciada pela tensão psíquica $e$ prematuridades oclusais 18 .

Sabe-se, também, que a duração dos contatos oclusars na deglutição é mais longa do que na mastigação $2 \mathrm{e}$, além disso, a direção das forças provenientes do impacto nas interferêr. cias de deslizamento é oblíqua ou mesmo horizontal, tendo um potencial para causar injúria traumática ao periodor:tium muito maior do que as forças aplicadas centralmente e na direção do longo do eixo dos dentes 18.

Embora tenha-se achado uma diferença entre as mobilidades dos dentes envolvidos nas interferências de deslizamento e seus homólogos e que esta diferença encontrada não ocorrè ao acaso uma vez que um teste estatístico foi feito a um nível de significância $p=0,01$ e daí resultou que a mesma era estatisticamente significante $(p<0,01)$, não se pode afirmar que a hipermobilidade dos dentes envolvidos seja patológica, já que, de acordo com Muhlemann 11 a mob:lidade patológica é causada por injúria nas estruturas periodontais e, para propósitos clín:cos, o limite superior da variação fisiológica da mobilidade horizontal dos dentes tem sido definida arbitrariamente como sendo de 15 centésimos de milímetro sob uma carga de 500 gramas.

$\mathrm{Na}$ amostra estudada, os limites de mobilidades fisiológicas não foram ultrapassados em nenhum dos casos. Este fato talvez possa ser explicado pelo fenômeno observado nos padrões eletromiográficos associados com con- 
tatos dentais chamado - "pausa motora", que é uma interrupção da atividade de contração muscular devida a mecanismos neuro-musculares ultrasensiveis. Assim, estes mecanismos podem evitar possiveis efeitos lesivos provenientes de contatos oclusais às expensas da resposta muscular autoprotetora 21.

Contudo, foi encontrada uma variabilidade elevada nas medições das mobilidades o que vem a confirmar as observações de estudos anteriores 4,20. Isso pode ser o resultado de erros de instrumentação, os quais são incontroláveis, enquanto que as outras var.áveis que poderiam influir desfavoravelmente na pesquisa foram postas sob controle pelas restrições que se impuseram para a aceitação dos participantes da mesma.

\section{CONCLUSÕES}

1. - As diferenças entre as mob:lidudes dos dentes envolvidos nas interferências de deslizamento $e$ seus homólogos eram, na amostra estudada, estatisticamente significante.

2. - Em nenhum dos casos foi ultrapassado o limite de mob:lidade fisiológica horizontal dos dentes estudados.

3. - Foi encontrada uma grande va. riabilidade nos valores das mobilidades horizontais.

\section{S U M Á R I O}

Uma adaptaçăo do macroperiodontômetro de Mühlemann foi utilizada em 36 estudantes com interferência de deslizamento.

As medições feitas nos dentes catalogados como interferências de desl.zamento e em seus homólogos indicaram que as mobilidades horizontais dos dentes envolvidos eram maiores do que as dos dentes controles. Contudo, os limites fisiológicos das mobilidades nunca foram ultrapassados. A análise dos resultados obtidos demonstrou que o sistema apresentava uma grande variab:lidade. 


\section{$-68-$ \\ REFERENCIAS BIBLIOGRAFICAS}

1. - GILLINGS, B. R. D., KOHL, J. T. and ZANDER, H. A.: Contact patterns using miniature radio transmitters. J. Dent. Res., 42: 177, 1963.

2. - GLICKMAN, I., PAMEIJER, J., ROEBER, F. and BRION, M.: Functional occlusion as revealed by miniaturized radio transmitters. Dent. Clin. N. Am. 13: 667, 1969.

3. - GRAF, H. and ZANDER, H. A.: Tooth contacts patterns in mastications. J. Prosth. Dent., 13: 1055, 1963.

4. - GREEN, E., CLAYTON, J. A. and SCHORK, M. A.: Longhudinal perlodontomeiry. J. Periodont. Res. 6: 301, 1971.

5. - HIRT, H. A. and MÜHLEMANN, H. R: Diagnosis of bruxism by means of tooth mobility measurements. Parodontologie, 9: 47, 1955.

6. - JANKELSON, B., HOFFMAN, G. M. and HENDRON, J. A.: Physiology of the stomatognathic 'system. J. Am. Dent. A., 46: 375, 1953.

7. - KERR, A. C., LEA, C. S. and MOODY, S.: A method of measuring the frequency of swallonwing in man. J. Dent. Res. 39: 668, 1960.

8. - MÜHLEMANN, H. R.: Periodontometry a method of measuring tooth mobility, Oral Surg., 4: 1220, 1951.

9. - MÜHLEMANN, H. R.: Tooth mobility v. tooth mobility changes through artificial trauma. J. Periodont., 25: 202, 1954.

10. - MÜHLEMANN, H. R.: Ten years of tooth-mobility measurements. J. Periodont., 31: 110, 1960.

11. - MÜHLEMANN, H. R.: Tooth mobility: a review of clinical aspects and research findings. J. Periodont., 38: 686, 1967.

12. - O'LEARY, R. J. and RUDD, K. D.: An instrument for measuring horizontal tooth mobility. Periodont., 1: 249, 1963.

13. - O'LEARY, T. J., RUDD, K, and NABERS, C.: Factors affecting horizontal tooth mobility. Periodontics, 4: 308, 1966.

14. - O'LEARY, T. J., RUDD, K.: NABERS, C. and STINUPF, T.: The effect of mastigation and deglutition on tooth mobility. Periodontics, 5: 26, 1967.

15. - O'LEARY, T. J., RUDD, K., NABERS, C. and STINUPF, A.: The effect of a "tube type" diet and stress-inducing conditions on tooth mobility. $J$. Periodont., 38: 222, 1967.

16. - O'LEARY, T. J.: Tooth mobility. Dent. Clin. N. Am., 13: 567, 1969.

17. - PARFITT, G. J.: Measurements of the physiological mobility of individual teeth in an axial direction. J. Dent. Res., 39: 608, 1960.

18. - RAMFJORD, S. P. and ASH, M. M.: Occlusion, 1970 2nd ed. Philadelphia, Saunders, 427 (p. 258).

19. - RATEITSCHAK, K. H.: Tooth mobility changes during pregnancy. J. Periodont. Res., 2: 199, 1967.

20. - RUDD, K. D., O'LEARY, T. J. and STUMPF, A. J.: Horizontal tooth mobility in carefully screeneo subjects. Periodont., 4: 65, 1964.

21. - SCHAERER, P., STALLARD, R. E. and ZANDER, H. A.: Oclusal interfe* rences and mastication: an electromyographic study. J. Prosth. Dent., 17: $438,1967$. 